\title{
Seismic behavior of a self-centering system with 2-4 viscous damper
}

N. K. Hazaveh, ${ }^{1}$ J.G. Chase ${ }^{2}$, G.W. Rodgers ${ }^{3}$, S. Pampanin ${ }^{4}$, R. Kordani ${ }^{5}$

\begin{abstract}
:
This research demonstrates the efficacy of a 2-4 viscous device in self-centering rocking structures, which are an emerging low damage seismic design approach in seismic zones. These systems have distinctly different dynamic response compared to the typically considered fixed base structures. In particular, they have bi-linear elastic response and, in this study, the results assess the relative impact of 2-4 devices versus typical viscous dampers and 1-3 viscous devices. Performance is assessed by maximum displacement $\left(\mathrm{S}_{\mathrm{d}}\right)$, total base shear $\left(\mathrm{V}_{\mathrm{b}}\right)$ and maximum acceleration $\left(\mathrm{S}_{\mathrm{a}}\right)$ indicative of structural, foundation and contents damage. Results show simultaneous reductions of displacement, base-shear and acceleration demands are only available with the 2-4 viscous device. Finally, a simple method is proposed to incorporate 2-4 viscous dampers into the design of self-centering systems using standard design approaches.
\end{abstract}

Keywords: 2-4 viscous damper, reducing base shear, self-centering structures, design procedure, Re-shaping hysteretic structural response

\footnotetext{
${ }^{1}$ Corresponding Author, PhD student, University of Canterbury, Christchurch, nikoo.hazaveh@pq.canterbury.ac.nz

${ }^{2}$ Distinguished Professor, University of Canterbury, Christchurch, geoff.chase@canterbury.ac.nz

${ }^{3}$ Associate Professor, University of Canterbury, Christchurch, geoff.rodgers@canterbury.ac.nz.

${ }^{4}$ Professor, University of Canterbury, Christchurch and Sapienza University of Rome stefano.pampanin@canterbury.ac.nz

${ }^{5}$ PhD student, University of Canterbury, Christchurch, reza.kordani@pg.canterbury.ac.nz
} 


\section{INTRODUCTION}

In the last decade, rocking mechanisms and self-centering have become well known as a structurally efficient and robust technology for seismic-resistant buildings [Priestley, 1991, Marriott et al., 2008, Marriott et al., 2009, Kam et al., 2010, Sarti et al., 2015]. These rocking systems have different dynamic response to a fixed base structure, due to the different response regimes that exist before and after the onset of rocking. Furthermore, due to the absence of sacrificial damage to the structural frame, rocking systems typically have low inherent damping. Therefore, to achieve adequate energy dissipation capacity under seismic excitations, various alternative energy dissipation elements (hysteretic, viscous or visco-elasto-plastic) need to be added in series and/or in parallel to self-centering systems [Marriott et al., 2008, Kam et al., 2010, Marriott et al., 2009, Rodgers et al., 2010, Mander et al., 2009, Rodgers et al., 2012].

Among supplemental dissipation devices, viscous dampers have been widely used in rocking wall and post-tensioned rocking bridge piers to improve the seismic behavior of these selfcentering system, as shown in Figure 1 [Marriott et al., 2008, Kam et al., 2010, Marriott et al., 2009]. Viscous dampers dissipate significant energy, but their reaction loads can increase foundation and overall base shear demands, reducing the ability to use them broadly in retrofit without significant added cost [Lin and Chopra, 2002, Hazaveh et al., 2016b, Filiatrault et al., 2001, Miyamoto and Singh, 2002, Vargas and Bruneau, 2007, Kam et al., 2010, Kam et al., 2008]. Thus, on the basis of a traditional performance-based seismic design and retrofit philosophy, designers are challenged by the difficult tradeoff between costs and acceptable damage (or targeted performance). 


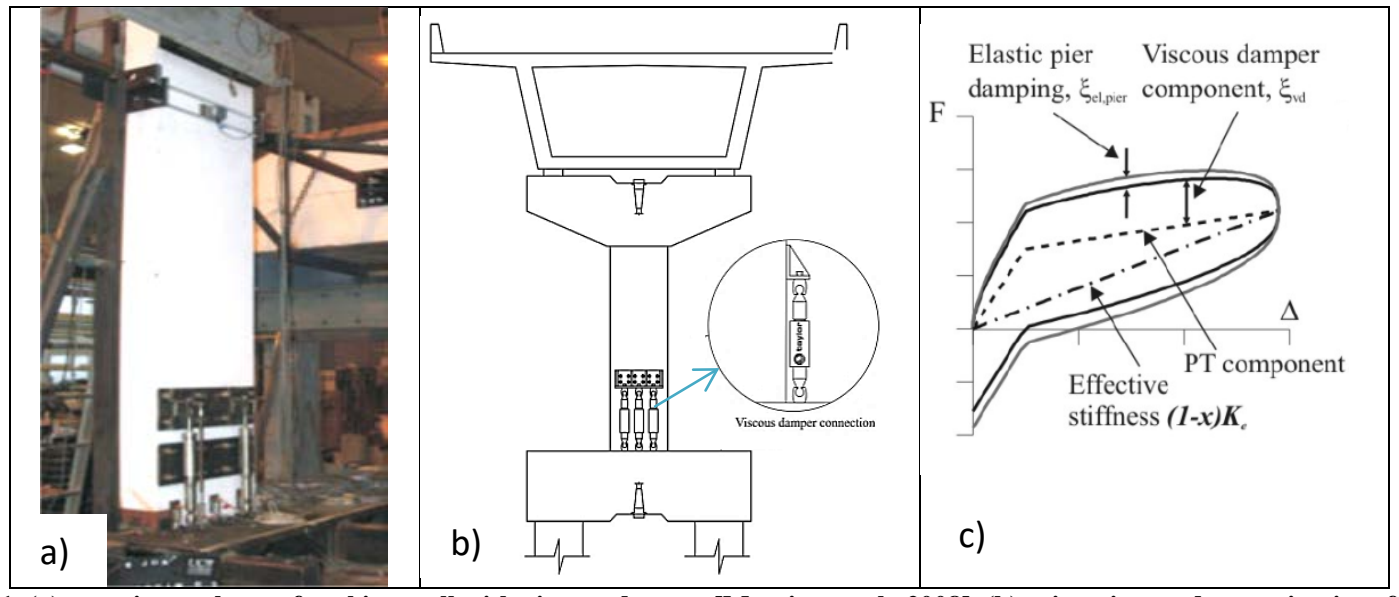

Figure 1. (a) experimental test of rocking wall with viscous damper [Marriott et al., 2008], (b) using viscous damper in pier of bridge [Marriott et al., 2008],(c) Decomposition of the transverse response of a post-tensioned bridge system with supplementary viscous [Marriott et al., 2008].

To address these issues, Hazaveh et al. [Hazaveh et al., 2016a, Hazaveh et al., 2016b, Hazaveh et al., 2015] introduced and examined two types of viscous dampers. Based on semi-active resettable stiffness devices [Mulligan et al., 2009, Rodgers et al., 2007] a 1-3 viscous damper provides resisting forces only in the first and third quadrants of the force-displacement plot. Similarly, a 2-4 viscous damper provides damping in the second and fourth quadrants. Spectral analysis shows typical viscous dampers increase the base shear of long period linear structures, typically greater than 2.7 sec [Hazaveh et al., 2016b]. However, adding a 2-4 viscous damper decreases base shear and displacement for all periods [Hazaveh et al., 2015, Hazaveh et al., 2016b, Mulligan et al., 2009, Rodgers et al., 2007]. The 2-4 device also has the potential benefit that it does not provide added forces during uplift, which can help to limit the total compression force applied to the toe of the wall during uplift, potentially reducing toe crushing during uplift of reinforced concrete rocking walls, while damping the return motion and re-seating of the wall.

However, the effect of the 2-4 viscous damper has only investigated on linear elastic structures [Hazaveh et al., 2015, Hazaveh et al., 2016b]. Therefore, there is a need to investigate the seismic behavior of self-centering system with these devices to validate this potential and growing area before application, including the need for design method to enable uptake. 
Hazaveh et al. [Hazaveh et al., 2016b] also suggested a method to calculate the damping reduction factor of linear structures with a 2-4 viscous damper. However, the resulting design procedure only considered linear elastic structures, and thus does not necessarily generalize to other structural systems, particularly those considering ductility. Therefore, to enable more widespread application of 2-4 viscous dampers in self-centering systems, suitable design procedures are needed. This potential is further enhanced by recent development of 1-3 and 24 viscous dampers that are entirely passive devices, and thus do not rely on complex semi-active control systems [Hazaveh et al., 2017].

This paper addresses these needs by evaluating the effect of typical, 1-3 and 2-4 viscous damping devices in self-centering SDOF rocking systems at a number of periods. The goal is to identify the range of potential reductions in displacement (structural demand), base shear (foundation demand) and acceleration (contents demand) with this type of device in comparison to a baseline case without supplemental damping. The analysis uses 60 earthquake ground motions from the SAC LA low, medium and high suites [Somerville and Venture, 1997]. The results would also indicate the distribution of possible reductions for ground motions with different probabilities of occurrence. Finally, this study uses these results to prepare a robust and simple design and analysis process to evaluate the effect of adding the 2-4 device to rocking structural systems that is a modified version of that linear system. 


\section{MODELING AND ANALYSIS METHODS}

This paper investigates the relative effectiveness of a traditional viscous damper, and the 1-3 and 2-4 viscous dampers on the seismic response of self-centering SDOF structural systems. Figure 2, illustrates the overall expected impact of three types of viscous dampers on the bilinear elastic structural response. The enclosed area is the energy dissipated per cycle due to supplemental damping. The self-centering rocking behavior is modeled numerically with an idealized bi-linear elastic spring [Priestley and Tao, 1993].

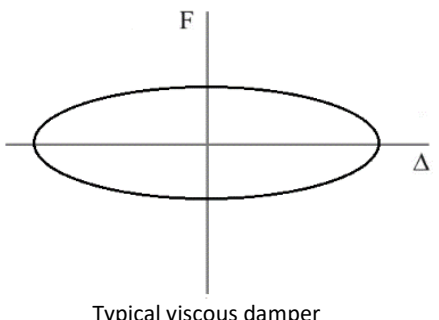

Typical viscous damper

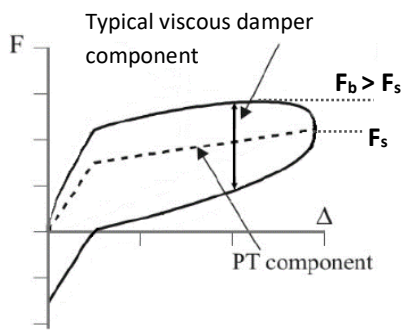

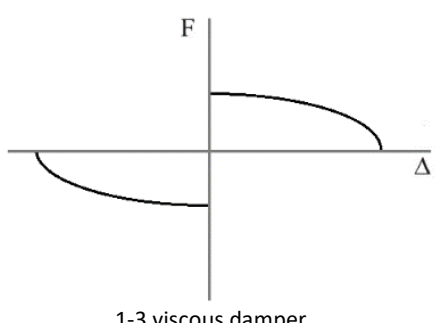

1-3 viscous damper

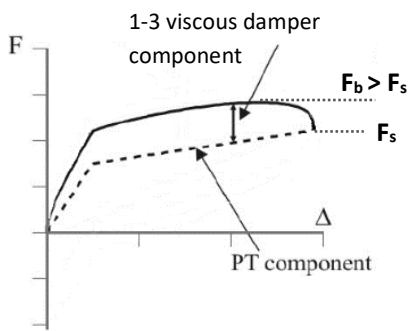

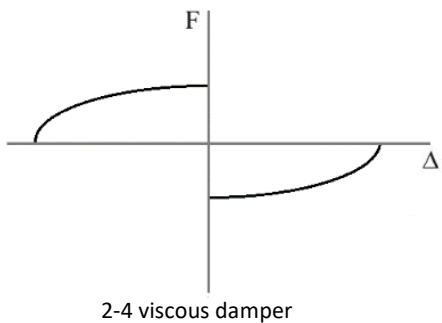

2-4 viscous damper

Figure 2. Schematic hysteresis for a typical, 1-3, and 2-4 viscous damper device, $F_{b}=$ total base shear, $F_{S}=$ base shear for undamped structure. Fb $>$ FS indicates an increase due to the additional damping.

The concept of force reduction factor $(R)$ and ductility $(\mu)$, which are fundamental tools in current seismic design are shown in Figure 3. For the equal displacement approximation, considered in this research, the displacement ductility factor is equal to the force-reduction factor [Priestley et al., 2007]:

$$
\mu=\frac{\Delta_{\max }}{\Delta_{y}}=R=\frac{F_{e l}}{F_{R}}
$$


where $F_{e l}$ is the maximum force developed at peak displacement , $\Delta_{\max }$, for a linear structure (labeled 1 in Figure 3) and $F_{R}$ is maximum force with force-reduction factor of $R$ at yielding displacement $\Delta_{y}$.(labeled 2 and 4 in Figure 3).

(a)

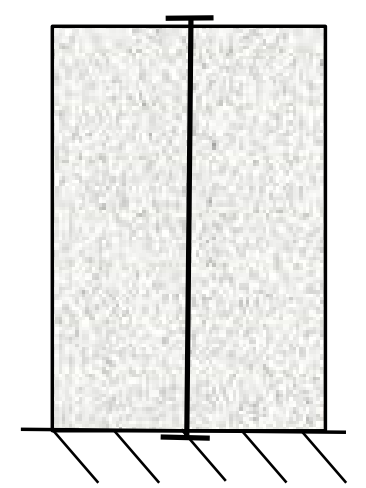

(b)

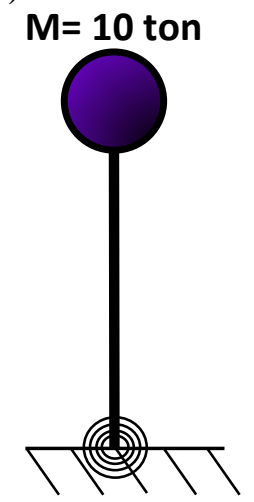

(c)

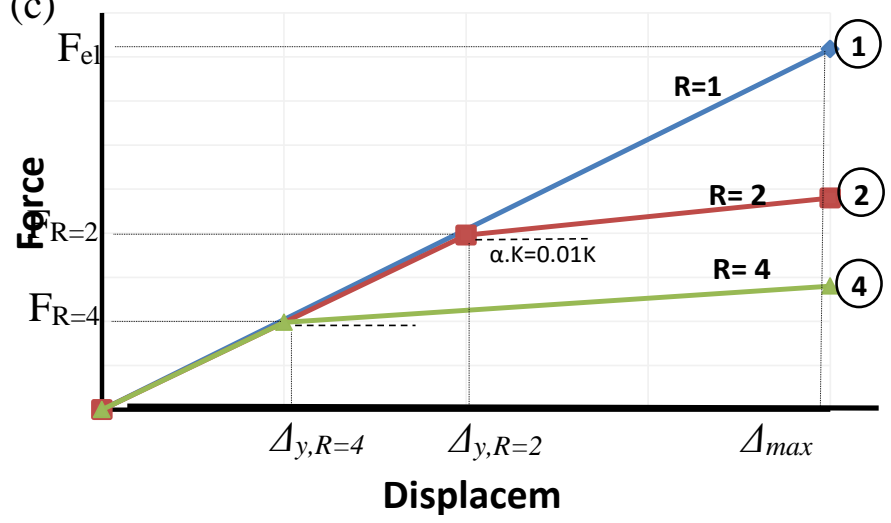

Figure 3. Prototype self-cantering wall; (b) simple SDOF representation; (c) The equal displacement approximation, the structure (1) is linear structure and the structure (2) and (4) have bilinear behaviour with ductility of 2 and 4, respectively.

In this study, bi-linear elastic structures with force design reduction factor (R) of 2.0 and 4.0, and period ranges of 0.5 to 4.5 s were considered for analysis. The structures were considered to be in Wellington on site class C soil [NZS1170, 2004]. Structures were designed to have same displacement as the code-specified spectral displacement of the site. Moreover, as the equal displacement approximation was verified for the structure considered in period ranges based on the time history analysis, the $\mathrm{R}$ values does not change the target displacement design. Figure 4 shows the basic design data for all 13 test cases covering this range of periods. The initial linear elastic stiffness is computed from the target period using a constant mass of $10000 \mathrm{~kg}$. Elastic displacement is calculated from dividing the design displacement by the desired force reduction (R) factor. The SDOF systems are taken to represent a prototype self-centering wall (Figure 3.ab), designed to a drift of $2 \%$ and the post-rocking stiffness is defined as $1 \%$ of the initial elastic stiffness. The range of case-study structures using the same $\mathrm{R}$ and $\mu$ in design creates a collection 
of 26 cases (13 periods times 2 different R-factors) to act like a traditional spectral analysis approach.

The model structures include inherent structural equivalent viscous damping of $5 \%$. The damping constant, $\mathrm{C}$, for each supplemental viscous damping device was determined based on a traditional viscous damper providing 15\% equivalent viscous damping. Thus, based on Figure 2, a 1-3 and 2-4 viscous damper will enclose approximately half the area of a traditional viscous damper. As such, the 1-3 and 2-4 devices will provide less equivalent viscous damping as they provide resistive forces for a smaller portion of the response cycle. The same $C$ value is then used for all supplemental viscous devices.

\begin{tabular}{ccc}
\hline $\begin{array}{c}\text { Period, } \\
\mathrm{T}(\mathrm{sec})\end{array}$ & $\begin{array}{c}\text { Design } \\
\text { Displacement } \\
\mathrm{S}_{\mathrm{d}}(\mathrm{T})[\mathrm{mm}]\end{array}$ & $\begin{array}{c}\text { Acceleration } \\
\text { design } \\
\text { coefficient } \\
\mathrm{C}_{\mathrm{d}}(\mathrm{T})[\mathrm{g}]\end{array}$ \\
\hline 0.1 & 2.9 & 1.17 \\
0.2 & 11.7 & 1.17 \\
0.3 & 26.1 & 1.17 \\
0.4 & 37.6 & 0.94 \\
0.5 & 49.7 & 0.80 \\
0.6 & 62.4 & 0.70 \\
0.7 & 75.7 & 0.63 \\
0.8 & 89.4 & 0.56 \\
0.9 & 103.6 & 0.51 \\
1 & 118.1 & 0.47 \\
1.5 & 196.3 & 0.36 \\
2 & 293.9 & 0.30 \\
2.5 & 406.7 & 0.26 \\
3 & 535.3 & 0.24 \\
3.5 & 582.6 & 0.19 \\
4 & 629.7 & 0.16 \\
4.5 & 653.4 & 0.13 \\
\hline
\end{tabular}

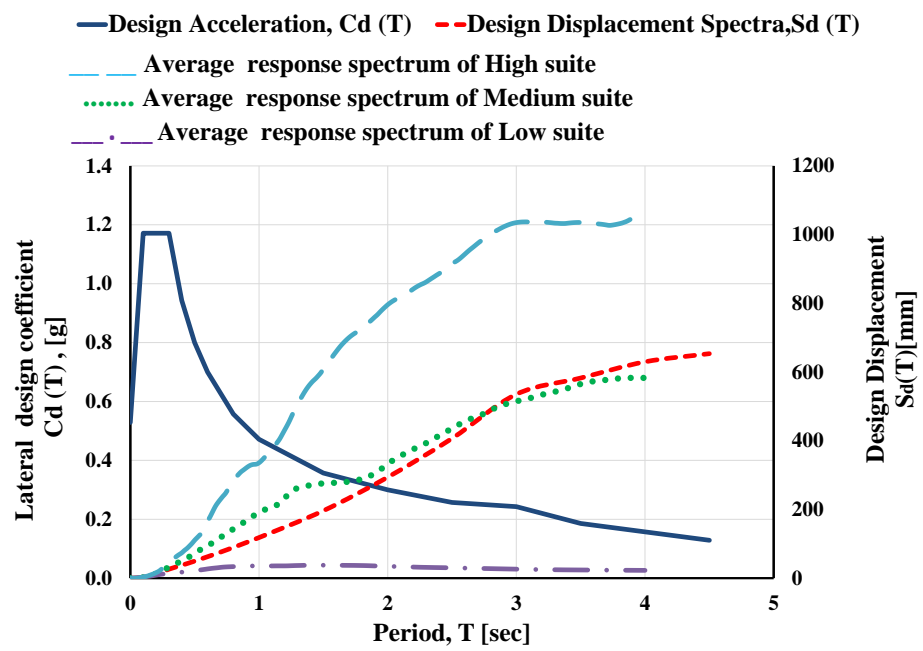

Figure 4. Elastic design displacement and acceleration spectra co-ordinates (5\% damped), Z=0.4, soil $\mathrm{C}, \mathrm{Sp}=1.0, \mathrm{D}<2 \mathrm{~km}[\mathrm{NZS1170}$ 2004] and average displacement response spectrum of low ,medium and high suit.

The analysis of each test structure utilizes 60 earthquakes from the 3 earthquake suites of the SAC project [Somerville and Venture, 1997]. Each suite is comprised of 10 different time histories with two orthogonal directions for each history. The 3 suites contain ground motions having probabilities of exceedance of 50\%, 10\% and 2\% in 50 years in the Los Angeles region, denoted the low, medium and high suites, respectively. The median spectral displacement of the records 
with a scale factor of unity is consistent with NZS1170.5 (2004) for Soil Type C Figure 4. The specific impact of near-fault events are not directly considered in this paper. However, the medium and high suites from the SAC project used in this research include several large nearfield events (e.g. Kobe and others), so these events are in the paper and part of the results. Thus, near field events are included but not specifically analysed as a subset. Using suites of ground motions, rather than a single individual event, eliminates the likelihood of erroneous conclusions due to variability in ground motions compared to structural dynamics. It is also allows risks of exceedance to be determined for specific probabilities of occurrence when comparing the impact of different devices or retrofits.

Reduction factors (RFs) for structural displacement $\left(\mathrm{S}_{\mathrm{d}}\right)$, base shear $\left(\mathrm{V}_{\mathrm{b}}\right)$ and acceleration $\left(\mathrm{S}_{\mathrm{a}}\right)$ demand are evaluated as a ratio to the baseline (no-device) case at the same level of $\mathrm{R}$ and structural period, for each ground motion. They specifically evaluate the range of potential reductions in response and associated risk of damage due to using these devices. These multiplicative RFs enable easy comparison of the different devices relative to the structural design case. Hence, the results can be applied to any sized structure, as they are only dependent on the device type, standard period, and damping of the device.

RFs less than 1.0 indicate a reduction in the response metric, while RFs greater than 1.0 indicate an increase in response. For each ground motion, RFs are determined, yielding 20 per suite. The median results is presented (50\% risk), but any level could be chosen. 


\section{RESULTS AND DISCUSSION}

Figure 5 shows the median structural displacement $\left(\mathrm{RF}_{\mathrm{Sd}}\right)$ and base shear $\left(\mathrm{RF}_{\mathrm{vb}}\right)$ reduction factors versus period for the 13 self-centering SDOF structures ( $T=0.5-4.5 \mathrm{sec}$ ) with $\mathrm{R}=2.0$ and 4.0, as shown in Figure 4. $\mathrm{RF}_{\mathrm{Sd}}$ is similar for $\mathrm{R}=2$ and 4, but results differ significantly in all cases for $\mathrm{RF}_{\mathrm{vb}}$. As expected, the typical viscous damper offers the greatest displacement reduction as it has the biggest area enclosed within the device hysteretic loop in Figure 2, but increases the overall base shear by the largest amount for almost all periods.

For example, for a period of $3.0 \mathrm{sec}, \mathrm{RF}_{\mathrm{vb}} \approx 3.0$ for the typical viscous device, indicating total base shear with the viscous damper is three times that of the uncontrolled (no device) case. Thus, while adding a viscous damper in linear structures increases base shear only for high periods above $2.7 \mathrm{sec}$ [Hazaveh et al., 2016b], for bi-linear systems the base shear increases for almost all periods. Similarly, the 1-3 device has $\mathrm{RF}_{\mathrm{Sd}}<1.0$ and $\mathrm{RF}_{\mathrm{vb}}>1.0$ for most periods. However, the 1-3 viscous device reduces displacement less than the 1-4 typical viscous damper as the area enclosed with the device hysteretic loop is approximately half the size, as shown in Figure 2. Only the low suite with $\mathrm{R}=2$ offers reduced displacement with $\mathrm{RF}_{\mathrm{vb}} \approx 1.0$, all other cases trade off reduced displacement with increased base shear for typical viscous damping and a 1-3 device.

In contrast, the 2-4 viscous device has $\mathrm{RF}_{\mathrm{Sd}}<1.0$ and $\mathrm{RF}_{\mathrm{vb}}<1.0$ in almost all cases. The exception is some select results with $\mathrm{RF}_{\mathrm{vb}}>1.0$, but by a much lesser amount than the 1-3 and typical viscous devices and only for the high-velocity excitations in the high suite ground motions. In these specific few cases, the damper resisting force in quadrants 2 and 4 exceeds the standard structural restoring forces in quadrants 1 and 3, resulting in an increase in the total base shear. For example, the total base shear of the structure with the period of $2.0 \mathrm{sec}$ and $\mathrm{R}=4$ 
under the LA38 earthquake with the 2-4 device increased for this reason, is illustrated in Figure 6. Hence, the 2-4 device offers reduced displacement and reduced base shear in all cases for all but the largest near field events with lowest probability of occurrence.
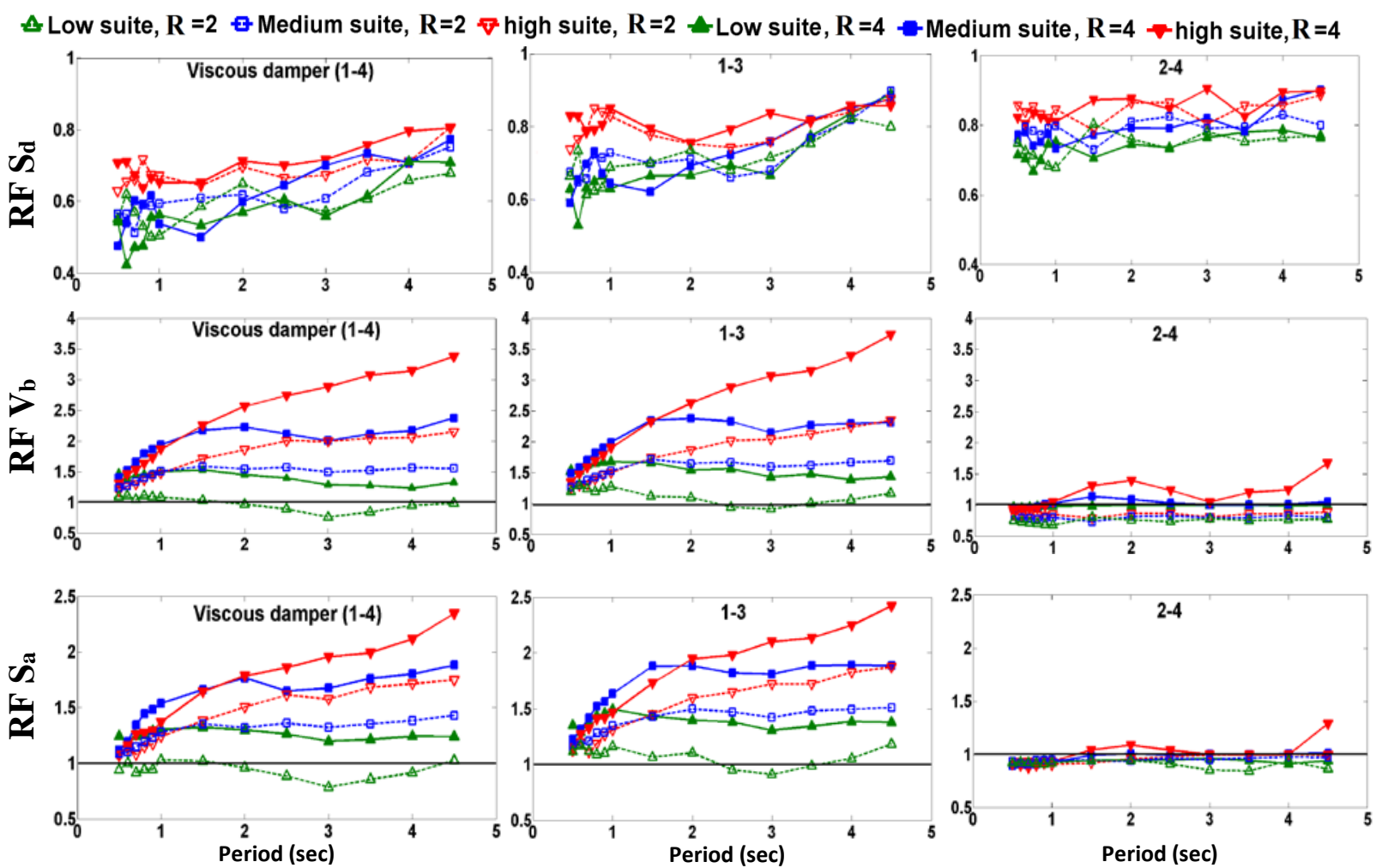

Figure 5. The median damping reduction factor of structural displacement, total base shear and acceleration of structures with periods $0.5 \mathrm{sec}$ to $4.5 \mathrm{sec}$ and $\mathrm{R}$ of 2.0 and 4.0 with three type viscous devices, with values of $5 \%$ additional damping under low, medium and high suite ground motion.

Overall, the 2-4 viscous device provides $\mathrm{RF}_{\mathrm{sd}}$ and $\mathrm{RF}_{\mathrm{vb}} \leq 1.0$ at levels that are relatively constant across periods. The 2-4 viscous damper approach thus offers the minimum variability in median level risk and thus the greatest robustness across structural periods, to a level not available from the other two devices considered. More specifically, the 2-4 viscous damper offers minimal risk of increased foundation demand along with reduced displacement demands. 




Figure 6. Force-displacement response of system with period of $2.0 \mathrm{sec}$ and ductility 4 under Palos Verdes earthquake (LA38, high Suite).

In addition, all the results in Figure 5 show the total base shear of the structures with $\mathrm{R}=4.0$ is greater than for $\mathrm{R}=2.0$ for all three types of viscous devices. This outcome can be explained by showing when any kind of viscous damper is added to the structure the $\mathrm{RF}_{\mathrm{vb}}$ for $\mathrm{R}=4.0$ is greater than $\mathrm{R}=2.0$ based on a short derivation:

$$
R F_{v b(R=2)}=\frac{F_{\text {with device }(R=2)}}{F_{\text {without device }(R=2)}}
$$

with considering the small post tensioning ratio (1\%) and the structural response is within the gap-opening regime (second section), the $\mathrm{RF}_{\mathrm{vb}}$ can be approximated as:

$$
R F_{v b(R=2)}=\frac{F_{\text {with device }(R=2)}}{F_{\text {without device }(R=2)}} \approx \frac{F_{\text {without device }(R=2)}+F_{\text {device }}}{F_{\text {without device }(R=2)}}
$$

And from Figure 3 and the definition of force reduction factor, R:

$$
F_{(R=4)} \approx \frac{1}{2} F_{(R=2)}
$$

The reduction factor for base shear for $\mathrm{R}=4.0$ can be computed similarly to that for $\mathrm{R}=2$ in Eq.3:

$$
R F_{v b(R=2)}=\frac{F_{\text {with device }(R=4)}}{F_{\text {without device }(R=4)}} \approx \frac{F_{\text {without device }(R=4)}+F_{\text {device }}}{F_{\text {without device }(R=4)}}
$$


Eq. 4 can be substituted into Eq. 5 to find the relationship between the RF of base shear for $\mathrm{R}=2$ and 4:

$$
\left.\begin{array}{rl}
R F_{v b(R=2)} \approx & \frac{F_{\text {without device }(R=2)}+F_{\text {device }}}{F_{\text {without device }(R=2)}} \\
R F_{v b(R=4)} \approx & \frac{F_{\text {without device }(R=2)}+2 F_{\text {device }}}{F_{\text {without device }(R=2)}}
\end{array}\right\} R F_{v b(R=2)}<R F_{v b(R=4)}
$$

The quantities used in Equations 2-6 and this outcome are illustrated graphically in Figure 7.

$\mathbf{R}=\mathbf{2}$

$\mathbf{R}=\mathbf{4}$
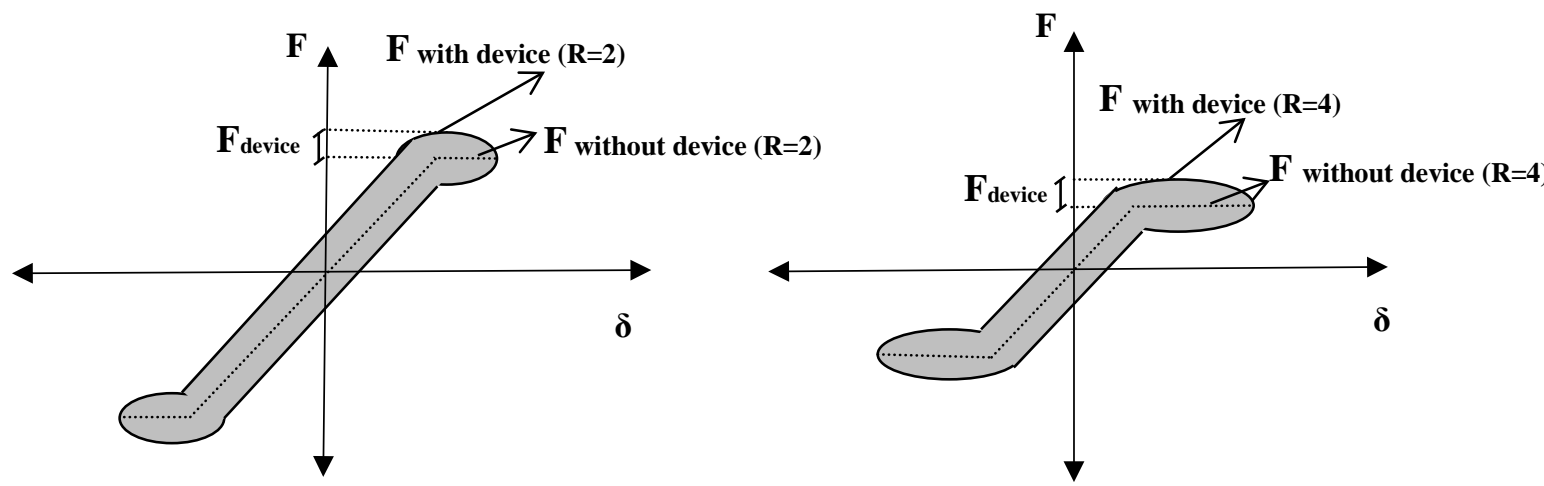

Figure 7. Force displacement of structures with $R=2$ and 4 and typical viscous damper

The median reduction factors for acceleration $\left(\mathrm{RF}_{\mathrm{Sa}}\right)$ results are also shown in Figure 5. Like those for total base shear, $\mathrm{RF}_{\mathrm{Sa}}>1.0$ for the 1-3 and typical viscous devices for most periods, and increase with structural period. However, $\mathrm{RF}_{\mathrm{Sa}}<1.0$ in most cases for the 2-4 device, providing further evidence of the efficiency of this approach.

To show more detail, the RF for $S_{d}, V_{b}$, and $S_{a}\left(R F_{S d}, R F_{V b}\right.$ and $\left.R F_{S a}\right)$ for the SDOF system with period $0.7 \mathrm{sec}$, with $\mathrm{R}=2.0$ and 4.0, are compared for all 3 devices and all 60 earthquakes in Figure 8. Figure 8 illustrates the tradeoffs in 3D of the different reduction factors. The box of $\mathrm{RF}_{\mathrm{Sd}}=\mathrm{RF}_{\mathrm{Vb}}=\mathrm{RF}_{\mathrm{Sa}}=1.0$ indicates the boundary between increased and decreased responses and results inside the box are thus desirable. Table 1 shows the number of cases within the box 
for each device summarizing all results for periods $\mathrm{T}=0.7,1.0$ and 1.5 seconds, by ground motion input suite.

The results in Table 1 indicate that only 7, 6, 7 and 9, 3, 5 cases of 120 are inside the box for the typical viscous and 1-3 devices for $\mathrm{T}=0.7,1.0$ and 1.5 seconds, respectively. All of them are structures with $\mathrm{R}=2$ under the low suite ground motion. In contrast, 111,81,86 of 120 cases for $\mathrm{T}=0.7,1.0$ and 1.5 are in the box for the 2-4 viscous damper and the others are from the much less likely to occure high suite. These results further support and quantify the outcomes presented. 


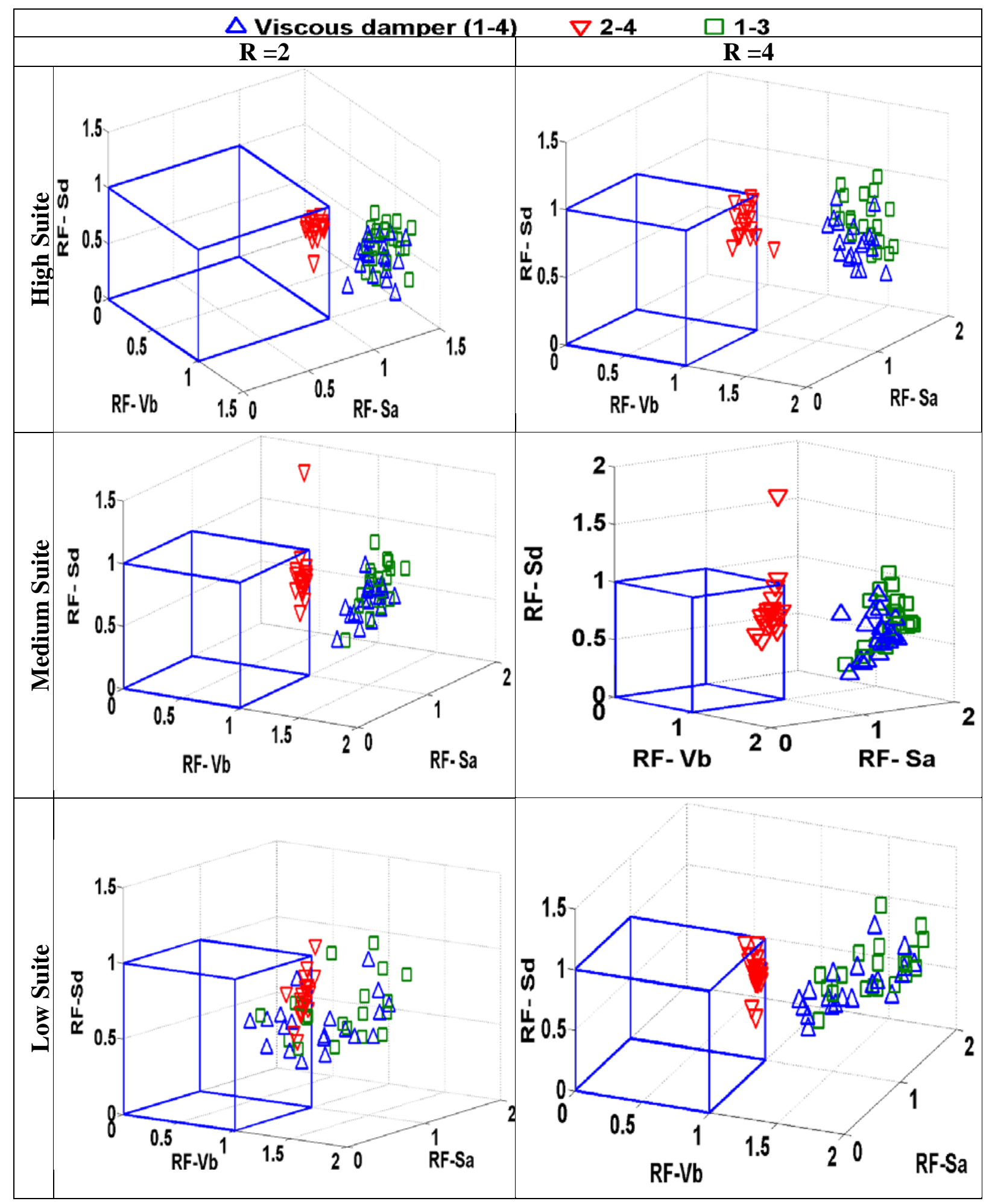

Figure 8. 3d Medium high low $\mathrm{R}=2$ and 4 at period $\mathrm{T}=0.7 \mathrm{sec}$. 
Table 1. No of cases in the box of Figure 8 that shows cases that RF of all of Sd, Vb and Sa are less than 1.0

\begin{tabular}{ccccc}
\hline $\begin{array}{c}\text { Period } \\
(\mathrm{sec})\end{array}$ & Device type & $\begin{array}{c}\mathrm{R}=2 \\
60 \text { Cases }\end{array}$ & $\begin{array}{c}\mathrm{R}=4 \\
60 \text { Cases }\end{array}$ & $\begin{array}{c}\text { Total result } \\
120 \text { cases }\end{array}$ \\
\hline \multirow{2}{*}{0.7} & Viscous (1-4) & 7 & 0 & 7 \\
& $1-3$ & 9 & 0 & 9 \\
& $2-4$ & 57 & 54 & 111 \\
\hline \multirow{2}{*}{1} & Viscous (1-4) & 6 & 0 & 6 \\
& $1-3$ & 3 & 0 & 3 \\
& $2-4$ & 57 & 29 & 86 \\
\hline \multirow{2}{*}{1.5} & Viscous (1-4) & 7 & 0 & 7 \\
& $1-3$ & 5 & 0 & 51 \\
\hline
\end{tabular}

\section{DESIGN AND ANALYSIS PROCEDURE}

In this section, a design and analysis procedure for rocking systems utilizing supplemental 2-4 viscous damping devices is described. Hazaveh et al. [Hazaveh et al., 2016a, Hazaveh et al., 2016b] suggested calculating the damping reduction factor of a structure with a 2-4 viscous damper by:

$$
\begin{cases}R F=\left(0.048\left(\xi_{0}+\xi\right)^{-0.5}-0.15\right) * T+0.9 & T \leq 2.7 \mathrm{sec} \\ R F=\left(\frac{0.07}{0.02+\left(\xi_{0}+\xi\right)}\right)^{0.22} & 2.7<T\end{cases}
$$

where $\xi_{0}$ represents the inherent elastic damping and $\xi$ is damping ratio provided by the 2-4 viscous device. Thus, for a targeted damping reduction factor $R F$, the required damping ratio for the device can be obtained by solving Eq.7, yielding [Hazaveh et al., 2016b]: 


$$
\begin{cases}\xi=\left(\frac{0.048 T}{R F-0.9+0.15 T}\right)^{2}-0.05 & T \leq 2.7 \mathrm{sec} \\ \xi=(0.07-0.02 \sqrt[0.22]{R F}) R F^{-4.54} & 2.7<T\end{cases}
$$

To use the Eq.7 and Eq.8 and reduce the number of variables of the rocking system, the system was simplified by representing the non-linear elastic post tensioned (PT) spring as an effective elastic spring with secant stiffness to the target displacement $\Delta_{d}$ [Priestley, 1991, Priestley and Tao, 1993, Priestley et al., 2007, Marriott et al., 2008]. This approach is illustrated in Figure 9, where $\mathrm{K}_{\mathrm{eff}}$ is the effective elastic stiffness of the post tensioned spring, and defined in Eq.9. The non-linear elastic post tensioned spring has been replaced with an equivalent elastic spring of stiffness $\mathrm{K}_{\mathrm{eff}}$, where the response of the viscous damper is not affected by the linearization of the PT spring. $K_{\text {eff }}$ is defined:

$$
K_{\text {eff }}=\frac{K(1+r \mu)}{\mu}
$$

where $K, r$ and $\mu$ is the initial stiffness, the ratio of post-rocking stiffness and ductility, respectively. The period of system using $\mathrm{K}_{\mathrm{eff}}$ is thus defined:

$$
T_{K_{e f f}}=2 \pi \sqrt{\frac{m}{K_{e f f}}}
$$




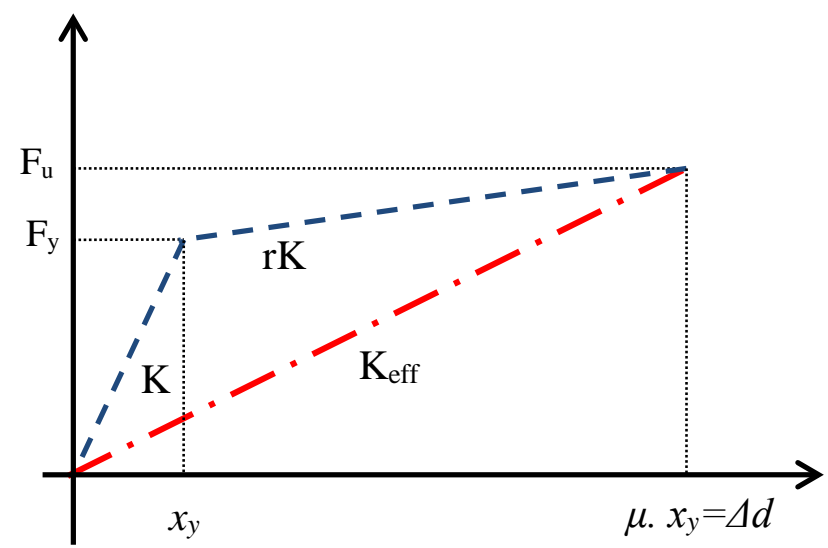

Figure 9. a) Equivalent displacement approximation, b) Effective stiffness $K_{\text {eff }}$

The period $\mathrm{T}_{\text {keff }}$ can be used in Eq.7 and 8 to find the damping reduction factor of the rocking system with the 2-4 viscous damper. Therefore, the damping reduction factor and required damping to obtain the desired RF for the rocking system can be computed:

$$
\begin{aligned}
& \left\{\begin{array}{lr}
R F=\left(0.048\left(\xi_{0}+\xi\right)^{-0.5}-0.15\right) * T_{K_{e f f}}+0.9 & T_{K_{e f f}} \leq 2.7 \mathrm{sec} \\
R F=\left(\frac{0.07}{0.02+\left(\xi_{0}+\xi\right)}\right)^{0.22} & 2.7<T_{K_{e f f}}
\end{array}\right. \\
& \left\{\begin{array}{lr}
\xi=\left(\frac{0.048 T}{R F-0.9+0.15 T_{K_{e f f}}}\right)^{2}-0.05 & T_{K_{e f f}} \leq 2.7 \mathrm{sec} \\
\xi=(0.07-0.02 \sqrt[0.22]{R F}) R F^{-4.54} & 2.7<T_{K_{e f f}}
\end{array}\right.
\end{aligned}
$$

Figure 10 provides a flowchart for calculating the RF of the bi-linear rocking system when a number, N, of 2-4 viscous dampers are added to the system. For example, three 2-4 viscous dampers, each with 5\% added damping, are installed to a structure with period of 1.0 sec, $125.66 \mathrm{kN} / \mathrm{m}$ stiffness, $1 \%$ the post-rocking stiffness and ductility 2.0. The effective stiffness and period is $63.46 \mathrm{kN} / \mathrm{m}$ (Eq.9) and $2.49 \mathrm{sec}$ (Eq.10), respectively. These values give a 
reduction factor of 0.79 (Eq.11). The actual damping reduction factor from simulation, directly calculated, is about 0.80 which is well estimated by proposed method (Figure 5, 10).

\section{Damping reduction factor determination for 2-4 viscous damper}

Adding N 2-4 viscous dampers in the rocking system

(e.g.: three 2-4 devices with $5 \%$ damping, $\mathrm{T}=1 \mathrm{sec}, \mathrm{r}=1 \%, \mathrm{~K}=125.66 \mathrm{kN} / \mathrm{m}, \mu=\mathrm{R}=2$ )

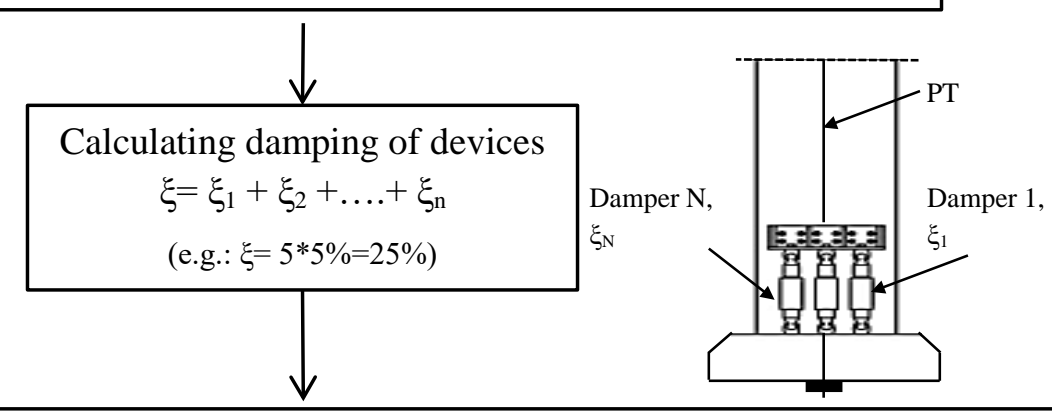

Calculating effective stiffness and period related to that

$$
\begin{array}{lc}
K_{\text {eff }}=\frac{K(1+r \mu)}{\mu} & \text { Eq.4-9 } \\
T_{K_{\text {eff }}=2 \pi \sqrt{\frac{m}{K_{\text {eff }}}}} & \text { Eq.4-10 } \\
\text { (e.g.: } \mathrm{K}_{\text {eff }}=63.4 \mathrm{kN} / \mathrm{m}, & \text { TKeff }_{\text {Ke }}=2.49 \mathrm{sec} \text { ) }
\end{array}
$$
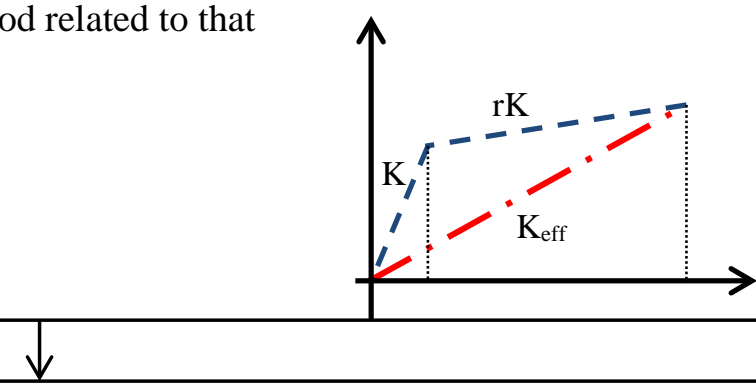

Calculating Reduction Factor (RF), Eq.4-11

$$
\begin{array}{cc}
R F=\left(0.048\left(\xi_{0}+\xi\right)^{-0.5}-0.15\right) * T_{K_{e f f}} & T_{K_{e f f}} \leq 2.7 \mathrm{sec} \\
R F=\left(\frac{0.07}{0.02+\left(\xi_{e l}+\xi\right)}\right)^{0.22} & 2.7<T_{K_{e f f}}
\end{array}
$$

(e.g.: $\mathrm{RF}=0.79)$

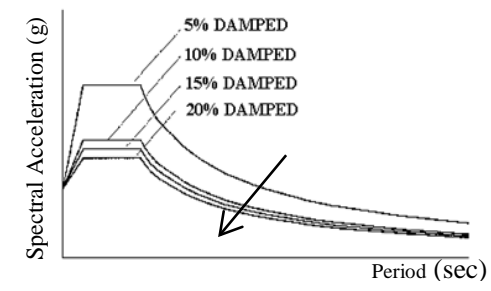

Figure 10. The flowchart for calculating the RF when the N 2-4 viscous dampers are added to the rocking system.

Figure 11 shows the flowchart for calculating the required size and number of 2-4 viscous dampers to achieve a desired, pre-specified damping reduction factor (RF). For instance, assuming that a 0.70 displacement damping reduction factor $\left(\mathrm{RF}_{\mathrm{sd}}\right)$ is desired for a structure with $\mathrm{T}=2.0 \mathrm{sec}, \mathrm{r}=1 \%, \mathrm{~K}=31.4 \mathrm{kN} / \mathrm{m}$ and $\mu=\mathrm{R}=2$ is desirable, then $33 \%$ (Eq.11) added damping 
is needed to have a $\mathrm{RF}_{\mathrm{sd}}=0.7$. Therefore, a device or devices with $\xi=35 \%$ damping is conservatively needed, which could be two devices with $17.5 \%$ added damping or a single device with 35\% added damping.

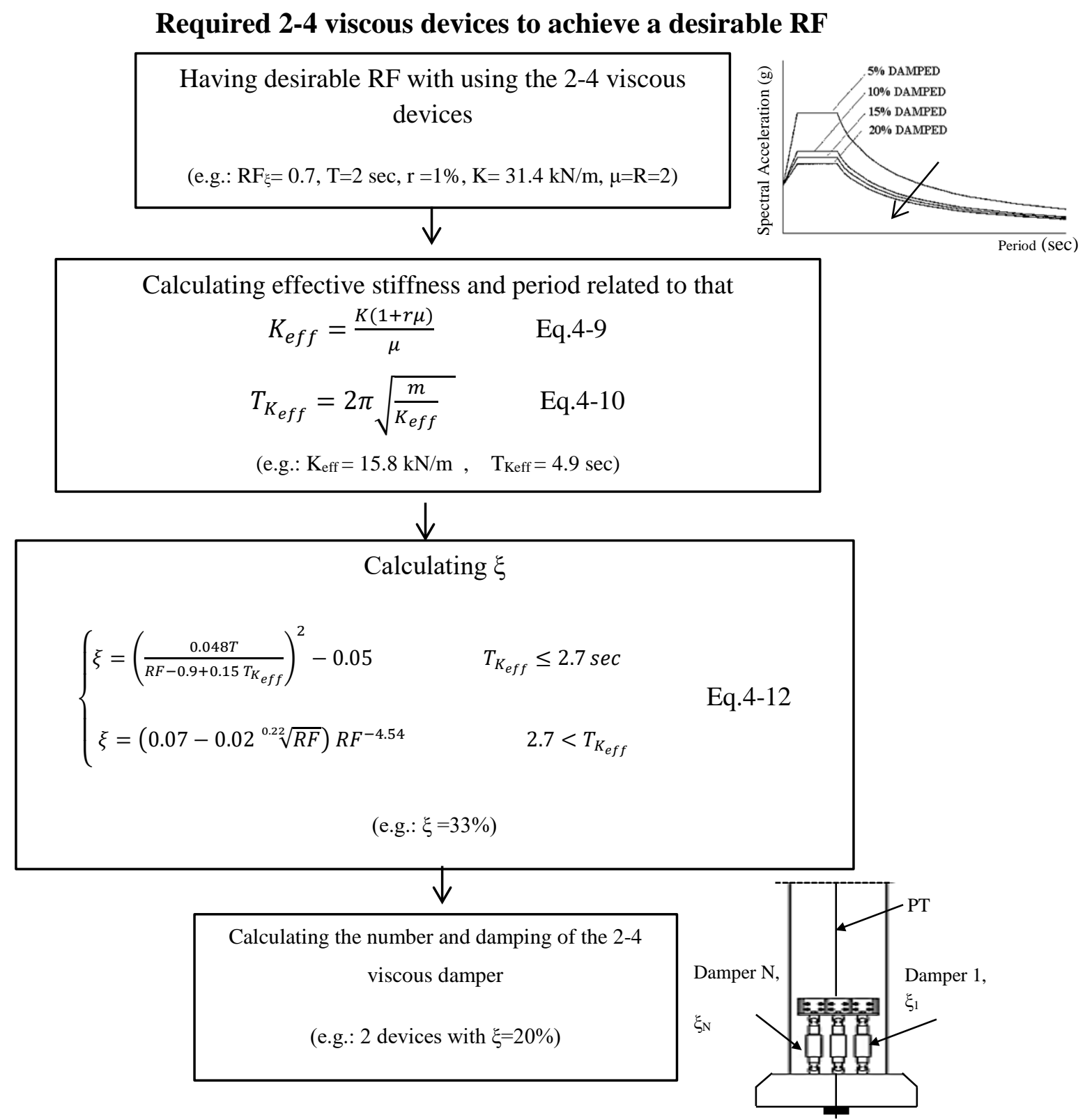

Figure 11 . Flowchart to find out the required dampers to have a desirable damping Reduction Factor. 


\section{CONCLUSIONS}

This study presents the analysis of using a novel 2-4 viscous damping device to re-shape structural hysteretic behavior to improve the seismic behavior of rocking structures using supplemental damping. Maximum displacement $\left(\mathrm{S}_{\mathrm{d}}\right)$, total base-shear $\left(\mathrm{V}_{\mathrm{b}}\right)$ and acceleration $\left(S_{a}\right)$ are calculated to determine the impact and efficiency of typical, 1-3 and 2-4 viscous damper on the seismic structural performance of bi-linear elastic rocking structures over a range of periods and ductility factors $\mathrm{R}=2$ and 4 . The main findings include:

1. The base shear increases for almost all periods in rocking system with the typical viscous damper by up to 3.5 times for high periods while in linear elastic structures adding viscous damper increases base shear only for longer periods, typically greater than 2.7s. Hence, greaters supplemental damping can be added to linear structures than bi-linear cases without penalty of increased base shear.

2. A 2-4 viscous damper can simultaneously reduce the values of displacement $\left(\mathrm{S}_{\mathrm{d}}\right)$, base shear $\left(\mathrm{V}_{\mathrm{b}}\right)$ and acceleration $\left(\mathrm{S}_{\mathrm{a}}\right)$ in all period ranges for bi-linear elastic systems under low and medium suite ground motions. For few cases with the high-velocity excitations in the high suite ground motion, applying a 2-4 viscous damper increases the base shear slightly, but the values were not as significant as the base shear of the structures with typical and 1-3 viscous damper. Hence, 2-4 damping devices provide a robust alternative with minimal to no penalty.

3. Displacement reduction factors, are similar for different design ductilities ( $\mathrm{R}=2$ and 4$)$. However, base shear, $\mathrm{RF}_{\mathrm{vb}}$, differs significantly in all cases for different ductility. In general, the total base shear and acceleration of the structures designed with higher ductility is greater than designed with lower ductility by approximately $50 \%$ for the 1 - 
3 and typical viscous devices. However, $\mathrm{RF}_{\mathrm{vb}}$ for the 2-4 devices is less dependent on ductility and provides more stable and constant behavior over different periods.

4. A simple method to determine the effect of the 2-4 device when added to new or existing bi-linear rocking systems and an overall design approach has been provided to enable more direct use of the results.

5. Installation of the proposed devices would be the same as for a typical viscous damper with similar limitations incurred in ensuring effective brackets and connections to ensure optimal load transfer between the device and the structure. However, since 2-4 viscous dampers can reduce displacement response without increasing base shear and floor accelerations, there is no added risk of increased foundation demand or damage, which could help minimize overall installation costs in comparison to typical viscous dampers and 1-3 viscous dampers, as seen in the results here.

These results indicate the robustness of simple 2-4 viscous dampers that can effectively mitigate seismic response of the self-centering system, and reduce the demand on the foundation and risk to contents and non-structural components.

\section{ACKNOWLEDGEMENT}

This project was (partially) supported by Quake CoRE, a New Zealand Tertiary Education Commission-funded Centre. Funding from the NZSEE/EQC Ivan Skinner award, the BRANZ Building Research Levy, and the Natural Hazards Research Platform is also gratefully acknowledged. 


\section{REFERENCE}

Filiatrault, A., Tremblay, R. \& Wanitkorkul, A. [2001] "Performance evaluation of passive damping systems for the seismic retrofit of steel moment-resisting frames subjected to near-field ground motions," Earthquake Spectra, 17, 427-456.

Hazaveh, N. K., Chase, J. G., Rodgers, G. W. \& Pampanin, S. [2015]."Control of Structural Response with a New Semi-Active Viscous Damping Device". 8th International Conference on Behavior of Steel Structures in Seismic Areas 2015.

Hazaveh, N. K., Rodgers, G. W., Chase, J. G. \& Pampanin, S. [2016a] "Reshaping Structural Hysteresis Response with Semi-active Viscous Damping," Bulletin of Earthquake Engineering 15, 1789-1806.

Hazaveh, N. K., Rodgers, G. W., Chase, J. G. \& Pampanin, S. [2017] "Experimental Test and Validation of a Direction and Displacement Dependent (D3) Viscous Damper," Journal of Engineering Mechanics,DOI: 10.1061/(ASCE)EM.1943-7889.0001354.

Hazaveh, N. K., Rodgers, G. W., Pampanin, S. \& Chase, J. G. [2016b] "Damping reduction factors and codebased design equation for structures using semi-active viscous dampers," Earthquake Engineering \& Structural Dynamics, 45, 2533-2550.

Kam, W., Pampanin, S., Palermo, A. \& Carr, A. [2008] "Implementation of advanced flag-shaped (AFS) systems for moment-resisting frame structures."

Kam, W. Y., Pampanin, S., Palermo, A. \& Carr, A. J. [2010] "Self-centering structural systems with combination of hysteretic and viscous energy dissipations," Earthquake Engineering \& Structural Dynamics, 39, 1083-1108.

Lin, W. H. \& Chopra, A. K. [2002] "Earthquake response of elastic SDF systems with non-linear fluid viscous dampers," Earthquake engineering \& structural dynamics, 31, 1623-1642.

Mander, T. J., Rodgers, G. W., Chase, J. G., Mander, J. B., MacRae, G. A. \& Dhakal, R. P. [2009] "Damage avoidance design steel beam-column moment connection using high-force-to-volume dissipators," Journal of structural engineering, 135, 1390-1397.

Marriott, D., Pampanin, S., Bull, D. \& Palermo, A. [2008] "Dynamic testing of precast, post-tensioned rocking wall systems with alternative dissipating solutions."

Marriott, D., Pampanin, S. \& Palermo, A. [2009] "Quasi-static and pseudo-dynamic testing of unbonded posttensioned rocking bridge piers with external replaceable dissipaters," Earthquake Engineering \& Structural Dynamics, 38, 331-354.

Miyamoto, H. K. \& Singh, J. [2002] "Performance of structures with passive energy dissipators," Earthquake spectra, 18, 105-119.

Mulligan, K., Chase, J., Mander, J., Rodgers, G., Elliott, R., Franco-Anaya, R. \& Carr, A. [2009] "Experimental validation of semi-active resetable actuators in a $1 / 5$ th scale test structure," Earthquake Engineering \& Structural Dynamics, 38, 517-536.

NZS1170 [2004] "NZS1170. 5: 2004," Structural Design Actions-Part, 5.

Priestley, M., Calvi, G. \& Kowalsky, M. [2007] Published. Direct displacement-based seismic design of structures. 2007 NZSEE Conference.

Priestley, M. N. [1991] "Overview of PRESSS research program," PCI journal, 36, 50-57.

Priestley, M. N. \& Tao, J. R. [1993] "Seismic response of precast prestressed concrete frames with partially debonded tendons," PCI Journal, 38, 58-69.

Rodgers, G. W., Mander, J. B., Geoffrey Chase, J., Mulligan, K. J., Deam, B. L. \& Carr, A. [2007] "Re-shaping hysteretic behaviour —spectral analysis and design equations for semi-active structures," Earthquake engineering \& structural dynamics, 36, 77-100.

Rodgers, G. W., Solberg, K. M., Mander, J. B., Chase, J. G., Bradley, B. A. \& Dhakal, R. P. [2010] "High-forceto-volume seismic dissipators embedded in a jointed precast concrete frame," Journal of Structural Engineering, 138, 375-386.

Rodgers, G.W., Mander, J.B. \& Chase, J.G. [2012] "Modeling Cyclic Loading Behavior of Jointed Precast Concrete Connections Including Effects of Friction, Tendon Yielding and Dampers" Earthquake Engineering \& Structural Dynamics (EESD), Vol 41(15), pp. 2215-2233, ISSN: 0098-8847. DOI: 10.1002/eqe.2183.

Sarti, F., Palermo, A. \& Pampanin, S. [2015] "Development and Testing of an Alternative Dissipative Posttensioned Rocking Timber Wall with Boundary Columns," Journal of Structural Engineering, 142, E4015011. Somerville, P. G. \& Venture, S. J. [1997]. Development of ground motion time histories for phase 2 of the FEMA/SAC steel project, SAC Joint Venture.

Vargas, R. \& Bruneau, M. [2007] "Effect of supplemental viscous damping on the seismic response of structural systems with metallic dampers," Journal of Structural Engineering, 133, 1434-1444. 
